\title{
Evidences for butterfly diagrams for spots on active late-type stars
}

\author{
Maria M. Katsova ${ }^{1}$, M.A.Livshits ${ }^{2}$ \\ and G.Belvedere ${ }^{3}$ \\ ${ }^{1}$ Sternberg State Astronomical Institute, Moscow State University, 119992 Moscow, Russia \\ email: maria@sai.msu.ru \\ ${ }^{2}$ IZMIRAN, 142190 Troitsk, Moscow Region, Russia email: livshits@izmiran.troitsk.ru \\ ${ }^{3}$ Department of Physics and Astronomy, University of Catania, Via Santa Sofia, 78 - 95125 \\ Catania, Italy, email: gbelvedere@ct.astro.it
}

\begin{abstract}
From long-term multicolour photometric observations of strongly spotted stars, one concludes usually on a presence of active longitudes. We argue the latitudinal effects in distribution spots over the surface and noticeable equatorward drift of the low-latitude boundary of the spotted region during the rising phase of activity.
\end{abstract}

\section{Introduction}

Application of inverse techniques and the Doppler imaging technique for analysis of photometric and spectral observations of active late-type stars gave a number of interesting results about stellar surface structures and, in particular, reasonable estimation of stellar spot area - filling factor $S_{\text {spot }}$. However, concerning the distribution of spots over the stellar surface, an opinion was established that "since a light curve represents a one-dimentional time series, the resulting stellar image contains information on the spot distribution only in one direction, in longitudes, while spot extents and location in latitudes remain uncertain" (Berdyugina et al. 2002). In fact, rotational modulation of brightness of a star is explained only by concentration of spots to one or a few active longitudes. This point of view seems to us not quite adequate to reality. Firstly, data on photometric variability through 30-40 years together with observations of rotational modulation contains information not only about active longitudes, but also about variations of latitudinal distribution of spots. Secondly, on the Sun latitudinal distribution of occurrence of solar spots is expressed clearly and their regular variations are observed as Maunder butterfly diagrams. From the other side, active longitudes on the Sun are pronounced weakly even at the descending phase of cycles, when this effect is maximal. Therefore, either we should consider the Sun as a unique star in the Universe or it needs to elaborate methods for the analysis of long-term stellar photometric variability.

\section{The butterfly diagrams for spots on active late-type stars}

We consider another case for the analysis of photometric data. Let's suppose that two spot belts exist in the northern and southern hemispheres and spots are concentrated slightly to an active longitude (figure 1). Such a model of the zonal spottedness was developed by Alekseev and Gershberg (1997), and references therein. New presentation of their results was given by us in two papers by Katsova et al. 2003, Livshits et al. 2003. We proposed a method in order to reveal a true analogue of the Maunder diagrams for 


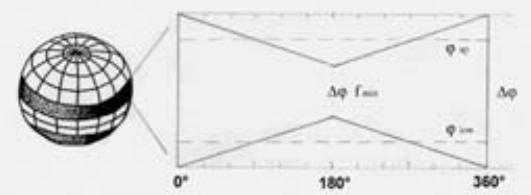

Shown on the left is the schema of the spot-belt boundaries. The minimal and maximal latitudinal extent ( $\Delta \varphi$ and $\Delta \varphi \times$ fmin ) of one of the spot belts of the zonal model is presented on a fragment of a synoptic map; the dashed lines are the upper and lower boundaries of the butterfly diagram for the spot distribution.

On the right:

the upper panel: temporal behaviour of the latitudes of occurence of spots (dashed lines); the butterfly diagram is labeled grey space between the solid lines; the lower panel: temporal behaviour of the relative spot area on LQ Hya.

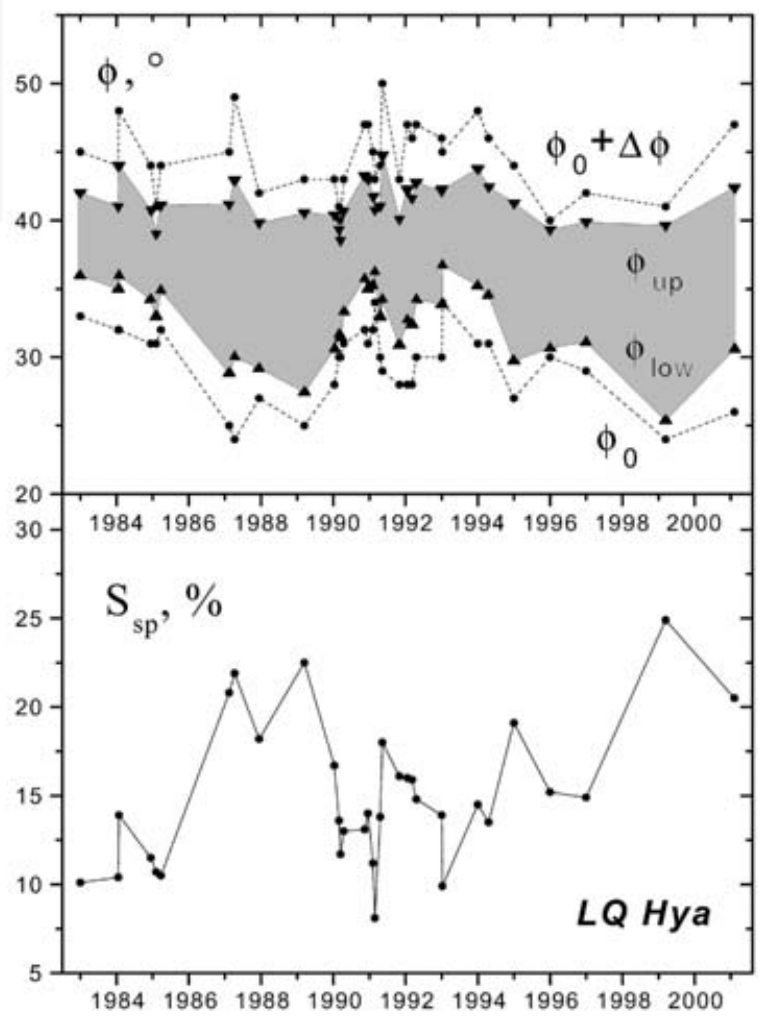

Figure 1. The butterfly diagram for spots on the LQ Hya

several strongly spotted late-type stars, which accordingly to the zonal models possess two spot belts. Results for the dK1e star LQ Hya are given in figure 1.

The main conclusion of our consideration is that the latitude of the lower boundary of the spot belts, $\phi_{\text {low }}$, decreases when the spot area $S_{\text {spot }}$ grows. It is traced for strongly spotted stars EK Dra, LQ Hya, VY Ari, and V775 Her, when both hemispheres are accessible to the observer and is practically absent for star V833 Tau, which is seen from the pole. As a rule the location of the upper boundary of the butterfly diagrams $\left(\phi_{\text {up }}\right)$ does not change. The rate of such an equatorward drift of the lower boundary is estimated as 1 to $2 \mathrm{deg} / \mathrm{yr}$ that is factor of $2-3$ smaller than the corresponding solar value.

We hope that the further development of this idea will help to reveal how spots are distributed over the stellar surface.

\section{Acknowledgements}

M.M.K. and M.A.L. would like to acknowledge the Organizing Committee for financial assistance for participation in this Symposium. M. M. K. is supported by RFBR under grant number 04-02-16068 and by Federal Program "Astronomy".

\section{References}

Alekseev, I. Yu. \& Gershberg, R. E. 1997 Astron. Rep. 40, 207.

Berdyugina, S. V., Pelt, J. \& Tuominen, I. 2002 Astron. \& Astrophys. 394, 505-515.

Katsova, M. M., Livshits, M. A. \& Belvedere, G. 2003 Solar Physics 216, 353-372.

Livshits, M.A., Alekseev, I.Yu. \& Katsova, M.M. 2003 Astron.Rep. 47, 562-572. 